\title{
Characterization of a novel variant of the second domain of bikunin with increased leukocyte elastase inhibitory activity
}

\author{
Masaki Nakamura*, Takashi Takeuchi, Yoshitaka Maeda, Yoshitaka Hosaka, Shoji Furusako \\ Discovery Research, Mochida Pharmaceutical Co., Ltd., Shizuoka, Japan; *Corresponding Author: mnakamur@mochida.co.jp
}

Received 13 December 2011; revised 29 January 2012; accepted 16 February 2012

\begin{abstract}
The light chain of inter- $\alpha$ inhibitor, also known as bikunin or urinary trypsin inhibitor, is composed of two tandemly arranged Kunitz-type protease inhibitor domains. The second domain of bikunin has factor $\mathrm{Xa}$ inhibitory activity which previously was enhanced by mutating two amino acids, glutamine 19 and tyrosine 46 to lysine and aspartate, respectively. In this study, we tried to potentiate its inhibitory activity against leukocyte elastase. A molecular docking model of the second domain of bikunin with leukocyte elastase revealed that P5 arginine 11 was a candidate residue for a third substitution. We generated six triple point mutants using site-directed mutagenesis, compared their leukocyte elastase-inhibitory activities, and selected the most potent variant with arginine 11 substituted to serine. The $\mathrm{IC}_{50}$ values for factor $\mathrm{Xla}$, factor $\mathrm{Xa}$, and leukocyte elastase were 182 , 302 , and $273 \mathrm{nM}$, respectively. Moreover, this triple point mutant prolonged the activated partial thromboplastin time and moderately reduced leukocyte elastase-induced endothelial injury. Additionally, favorable conformations created by these mutations were speculated using the structure of the Kunitz protease inhibitor domain of protease nexin 2 complexed with factor Xla as a reference. We discovered a novel triple point mutant of the second domain of bikunin that has potent inhibitory activities against factor Xla, factor $\mathrm{Xa}$, and leukocyte elastase. This variant exhibited anticoagulant activity in plasma and suppressed endothelial cell injury.
\end{abstract}

Keywords: Inter- $\alpha$ Inhibitor; Bikunin; Kunitz-Type Protease Inhibitor; Leukocyte Elastase; Factor Xla

\section{INTRODUCTION}

Coagulation and inflammation are closely related pro- cesses and serine proteases have several roles in these cascades [1]. Systemic inflammation potently stimulates coagulation by upregulating tissue factor and downregulating natural anticoagulants such as antithrombin. The tissue factor-factor VIIa complex activates factor X, which is a key molecule in the coagulation cascade and is responsible for thrombin generation [2]. Thrombin not only generates fibrin clots but also amplifies the intrinsic coagulation cascade by activating factor XI. It is well known that factor XIa is also important in the coagulation cascades. Factor XI-deficient animals are resistant to pathologic intravascular thrombus formation [3] and coagulopathy during sepsis [4]. Moreover, thrombin initiates an inflammatory response by activating protease-activated receptors, leading to endothelial dysfunction [5]. On the other hand, leukocyte elastase is released from activated neutrophils and destroys antithrombin, C1-esterase inhibitor, thrombomodulin, and tissue factor pathway inhibitor, leading to amplified procoagulant responses [6]. Furthermore, leukocyte elastase degrades almost all components of the extracellular matrix, leading to tissue injury [7]. Therefore, agents that can inhibit leukocyte elastase as well as activated coagulation factors including factor XIa and Xa may be of great therapeutic value to treat pathologic conditions such as disseminated intravascular coagulation and acute lung injury.

The light chain of inter-inhibitor, also known as bikunin, urinary trypsin inhibitor (UTI), or ulinastatin, is a 24.3 $\mathrm{kDa}$ glycoprotein [8] that is responsible for the serine protease inhibitory activity of inter-inhibitor $[9,10]$. The structure of bikunin is composed of two tandemly arranged Kunitz-type protease inhibitor domains connected by five amino acids and the trypsin and leukocyte elastase inhibitory activities exist in the second domain [11]. Additionally, analysis of the crystal structure of bikunin revealed that these Kunitz-type domains are very similar to other proteins with this fold [12] including Kunitz protease inhibitor domain of protease nexin 2 which inhibits coagulation factors. Bikunin is isolated from human urine and administered as a treatment for acute pan- 
creatitis, chronic relapsing pancreatitis, and acute circulatory failure or shock in Japan. It has been believed that bikunin exhibits therapeutic benefits because of its broad spectrum of serine protease inhibitory activities. However, the inhibitory activities against respective proteases are still moderate. Therefore, there remains a need to enhance the protease inhibitory activities of bikunin and improve its efficacy.

To obtain a more potent serine protease inhibitor, Morishita $e t$ al. expressed and purified the second domain of bikunin, encoding 70-amino-acid residues from threonine 78 to the C-terminal asparagine (Figure 1) [13]. This recombinant second domain of bikunin could inhibit factor $\mathrm{Xa}$ and plasma kallikrein more potently than bikunin. However, the inhibitory activity against leukocyte elastase was approximately 2-times less potent than that of bikunin. Furthermore, Nii et al. theoretically modeled the structure of the second domain of bikunin complexed with factor Xa based on the X-ray crystal structure of bovine pancreatic trypsin inhibitor bound to bovine pancreatic trypsin. Using this information, the factor $\mathrm{Xa}$ inhibitory activity of the second domain of bikunin was potentiated by genetically mutating two amino acids, glutamine 19 and tyrosine 46 to lysine and aspartate, respectively [14]. However, it is still unknown which amino acid substitution in the second domain could recover or increase the inhibitory activity against leukocyte elastase.

Coagulation factors, such as factor XIa and factor Xa, and leukocyte elastase belong to the chymotrypsin family of serine proteases, which share more than $40 \%$ sequence

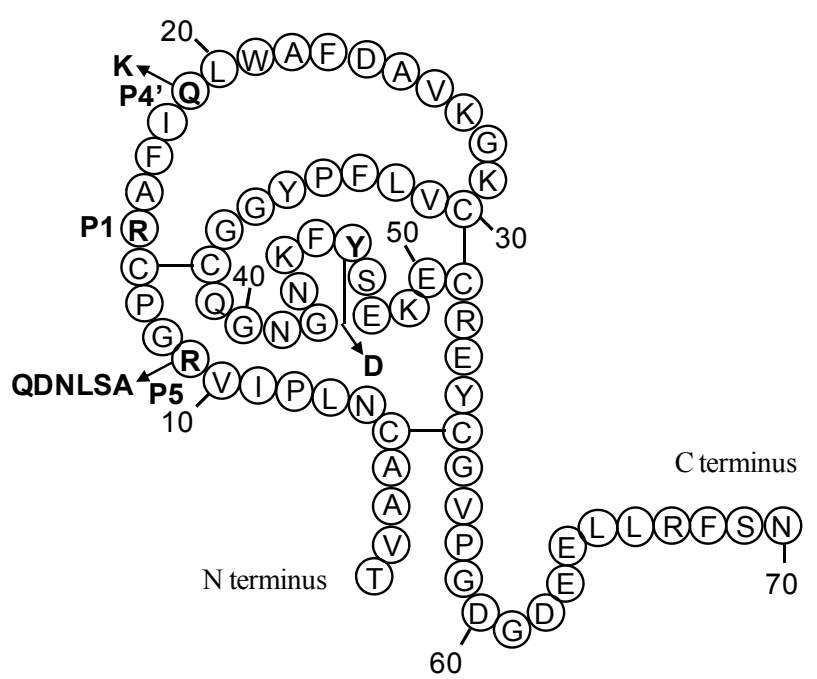

Figure 1. The primary structure of the second domain of bikunin and its variants. Amino acid numbering was started at the $\mathrm{N}$-terminus of the second domain. P5 arginine 11 was substituted to glutamine, aspartate, asparagine, leucine, serine, or alanine. P4' glutamine 19 was substituted to lysine. Tyrosine 46 was substituted to aspartate. Amino acids are indicated by their single-letter code. Mutated residues are indicated by arrows and reactive site residues, $\mathrm{P} 1$ and $\mathrm{P} 5$, are labeled. homology in their active sites and have similar catalytic mechanisms $[15,16]$. Moreover, both coagulation factors and leukocyte elastase are inhibited by common inhibitor classes, including isocoumarins [17] and bikunin [9,11]. Therefore, we speculated that we could design a novel derivative of the second domain of bikunin that inhibits multiple enzymes, such as coagulation factors and leukocyte elastase, based on the structural homology of their active sites. In the present study, we tried to potentiate the leukocyte elastase inhibitory activity of the second domain of bikunin by amino acid substitution without losing anticoagulant activity. To search amino acid residues to be modified, we examined a theoretical model of the second domain of bikunin docked to the X-ray structure of human leukocyte elastase. Using this information, we generated a novel triple mutant that inhibits factor XIa, factor Xa, and leukocyte elastase. In addition, we constructed models of this novel variant as a complex with serine proteases using the crystal structure of the Kunitz protease inhibitor domain of protease nexin 2 complexed with human factor XIa as a reference to identify novel interactions.

\section{MATERIALS AND METHODS}

\subsection{Reagents and Proteins}

The restriction enzymes and modifying enzymes used in this study were purchased from Takara Bio Inc. (Japan) or Toyobo Co., Ltd. (Japan). E. coli strains HB101 and JE5505 were used for recombination and gene expression, respectively. Linkers for site-directed mutagenesis, HindIII primer (5'-ACGGAAGTTCACGTAAAA AGC-3') and pBR BamHI primer (5'-ACGATGCGTTCCGGCG TAGAG-3') were synthesized with a DNA synthesizer (Model 381A, Applied Biosystems Inc., USA). Purified human serine proteases, factor XIa, factor IXa, factor $\mathrm{XIIa}$, and activated protein $\mathrm{C}$ were purchased from American Diagnostica Inc. (USA); factor Xa and factor VIIa from Enzyme Research Laboratories Inc. (USA); thrombin and plasmin from Sigma-Aldrich Co. (USA); leukocyte elastase and cathepsin G from Athens Research \& Technology Inc. (USA); plasma kallikrein from Calbiochem (USA); tissue-type plasminogen activator from Kyowa Hakko Kogyo Co., Ltd. (Japan); and urokinase-type plasminogen activator from Kowa Company, Ltd. (Japan). Synthetic peptide substrates for serine proteases, S-2366, S-2222, S-2238, S-2288, S-2484, S2302, and S-2251 were purchased from Chromogenix AB (Sweden); SPECTROZYME FIXa and SPECTRO ZYME FXIIa from American Diagnostica Inc. (USA); and Cathepsin G substrate III from Calbiochem (USA). Bikunin also known as urinary trypsin inhibitor, the second domain of bikunin without $\mathrm{N}$-terminal two amino acids (abbreviated as D2 in the present paper), and D2 with 
glutamine 19 substituted to lysine/tyrosine 46 substituted to aspartate (abbreviated as D2(Q19K/Y46D) in the present paper) were prepared as described previously $[13,14]$.

\subsection{Docking Study of the Second Domain of Bikunin with Human Leukocyte Elastase}

To construct a docked structure of the second domain of bikunin with human leukocyte elastase, the X-ray crystal structure of bovine pancreatic trypsin inhibitor (BPTI) complexed with bovine trypsin (Protein Data Bank (PDB) ID 2PTC) was used as a template [18]. After the X-ray structure of human leukocyte elastase (PDB ID 1HNE) was superimposed onto bovine trypsin (2PTC) at the structurally conserved regions (SCRs) among the serine proteases, coordinates of the second domain of bikunin were generated by replacing the side chains of BPTI. Construction of the docked structure and energy minimization of the structure were performed with BIOCES[E] system version 1.4 (NEC, Tokyo) as described by Miyata et al. [19].

\subsection{Site-Directed Mutagenesis}

The primary structure of second domain of bikunin is illustrated in Figure 1. Site-directed mutagenesis of D2 $(\mathrm{Q} 19 \mathrm{~K} / \mathrm{Y} 46 \mathrm{D})$ was performed as previously reported [14]. Plasmid pM594 [13] was used as a template after the nucleotides coding for 12 -amino acid sequence, A-VL-P-Q-E-E-E-G-S-G-M, were inserted between phoA signal peptide and D2(Q19K/Y46D). A 25- to 27-base oligomer with 12 to 13 bases flanking both the 5'- and 3 '-sides of the one to three nucleotides to be substituted was synthesized as a linker for the site-directed mutagenesis. A mutation was introduced as described previously [20]. To substitute another amino acid in D2(Q19K/Y46D), the first polymerase chain reaction (PCR) was performed with the linker and HindIII primer, and the resulting PCR product and $\mathrm{pBR}$ BamHI primer were used for the second PCR. The obtained DNA fragment was digested with HindIII/BamHI and inserted into the HindIII/BamHI sites of pM594. The nucleotide sequences of the HindIII/BamHI fragments containing modified second domain DNAs in the variant-expression plasmids were verified by the M13 dideoxy termination method [21]. Expression of the genes, confirmation of the purified products by SDS-PAGE, and measurement of protein concentrations were performed as previously described $[13,14]$. For purification of the expressed variants, collected inclusion bodies were solubilized with guanidine hydrochloride and then renatured with glutathione. After dialysis against distilled water, the renatured variants were treated by cyanogen bromide and subsequently purified using a Mono S cation-exchange column (GE
Healthcare, USA).

\subsection{Measurement of Serine Protease Activities}

The inhibition of various serine proteases was examined using peptide-p-nitroanilide substrates in flat-bottom 96-well microtiter plates. An enzyme solution and the test sample dissolved in physiological saline were added to a buffer $(140 \mathrm{mM} \mathrm{NaCl}, 5 \mathrm{mM} \mathrm{CaCl} 2,0.1 \%$ bovine serum albumin and $20 \mathrm{mM}$ Tris- $\mathrm{HCl}, \mathrm{pH}$ 7.4) and preincubated at $37^{\circ} \mathrm{C}$ for $5-10 \mathrm{~min}$. Then, an appropriate substrate was added, and the reaction mixture was incubated at $37^{\circ} \mathrm{C}$. The reaction was stopped by the addition of acetate solution and absorbance at $405 \mathrm{~nm}$ was measured. Final concentrations of proteases and substrates were chosen to yield similar substrate hydrolysis rates as described in Table 1. The 50\% inhibitory concentrations $\left(\mathrm{IC}_{50}\right)$ were determined by fitting non-linearly a sigmoidal equation to a dose-response curve using Prism software (GraphPad, USA).

\subsection{Anticoagulant Activity in Human Plasma}

Activated partial thromboplastin time was measured using an automatic coagulometer (AMAX CS-190, Amelung $\mathrm{GmBH}$, Germany). Pooled human plasma (CiTrol ${ }^{\circledR}$, Coagulation Control, level 1) was purchased from Dade Behring Inc. (USA). One hundred thirteen microliters of plasma and $20 \mathrm{~L}$ of the test sample dissolved in physiological saline were mixed. Then, $50 \mathrm{~L}$ of the mixture and $50 \mathrm{~L}$ of activated partial thromboplastin time reagent $\left(\right.$ Actin $^{\circledR}$, Activated Cephaloplastin Reagent, Dade Behring Inc., USA) were added to the assay cuvette and incubated at $37^{\circ} \mathrm{C}$ for $2 \mathrm{~min}$. Fifty microliters of $25 \mathrm{mM}$ $\mathrm{CaCl}_{2}$ solution was added to initiate the reaction.

\subsection{Leukocyte Elastase-Induced Endothelial Injury}

Human umbilical vein endothelial cells (Lonza Group Ltd., Switzerland) were seeded (20,000 cells/well) in 96well culture plates and incubated overnight in RPMI1640 containing $10 \%$ human serum. Cells were then incubated with the test sample in the presence or absence of human leukocyte elastase $(0.3 \mathrm{~g} / \mathrm{mL})$ for $2 \mathrm{~h}$ at $37^{\circ} \mathrm{C}$ in a $5 \% \mathrm{CO}_{2}$ atmosphere. Cell viability was determined by measuring the absorbance at $450 \mathrm{~nm}$ using WST-1 reagent (Takara Bio Co., Ltd., Japan) according to the manufacturer's instructions.

\subsection{Comparative Modeling of Complexes between the Novel Variant and Serine Proteases}

Sequences of Kunitz protease inhibitor domains were 
Table 1. Conditions of serine protease assays.

\begin{tabular}{ccc}
\hline Human enzyme (final concentration) & Substrate (final concentration) & $30 \mathrm{~min}$ \\
\hline Factor XIa $(0.02 \mathrm{U} / \mathrm{mL})$ & $\mathrm{S}-2366(0.5 \mathrm{mM})$ & $30 \mathrm{~min}$ \\
Factor Xa $(0.02 \mathrm{U} / \mathrm{mL})$ & $\mathrm{S}-2222(0.4 \mathrm{mM})$ & $20 \mathrm{~min}$ \\
Thrombin $(0.1 \mathrm{U} / \mathrm{mL})$ & $\mathrm{S}-2238(0.4 \mathrm{mM})$ & $120 \mathrm{~min}$ \\
Factor VIIa $(5 \mu \mathrm{g} / \mathrm{mL})$ & $\mathrm{S}-2288(0.8 \mathrm{mM})$ & $30 \mathrm{~min}$ \\
Factor IXa $(5 \mu \mathrm{g} / \mathrm{mL})$ & SPECTROZYME FIXa $(1 \mathrm{mM})$ & $10 \mathrm{~min}$ \\
Factor XIIa $(0.3 \mathrm{U} / \mathrm{mL})$ & SPECTROZYME FXIIa $(0.5 \mathrm{mM})$ & $5 \mathrm{~min}$ \\
Leukocyte elastase $(2 \mu \mathrm{g} / \mathrm{mL})$ & S-2484 $(0.2 \mathrm{mM})$ & $6 \mathrm{~h}$ \\
Cathepsin G $(10 \mu \mathrm{g} / \mathrm{mL})$ & Cathepsin G substrate III $(1 \mathrm{mM})$ & $30 \mathrm{~min}$ \\
Plasma kallikrein $(0.1 \mu \mathrm{g} / \mathrm{mL})$ & $\mathrm{S}-2302(0.4 \mathrm{mM})$ & $60 \mathrm{~min}$ \\
Activated protein C $(100 \mathrm{ng} / \mathrm{mL})$ & $\mathrm{S}-2366(0.4 \mathrm{mM})$ & $40 \mathrm{~min}$ \\
t-PA $(1000 \mathrm{IU} / \mathrm{mL})$ & $\mathrm{S}-2288(0.8 \mathrm{mM})$ & $20 \mathrm{~min}$ \\
u-PA $(600 \mathrm{U} / \mathrm{mL})$ & $\mathrm{S}-2222(0.4 \mathrm{mM})$ & $20 \mathrm{~min}$ \\
Plasmin $(0.01 \mathrm{U} / \mathrm{mL})$ & $\mathrm{S}-2251(0.4 \mathrm{mM})$ & $20 \mathrm{~min}{ }^{\mathrm{a})}$ \\
Leukocyte elastase $(1 \mu \mathrm{g} / \mathrm{mL})$ & $\mathrm{STANA}(5 \mathrm{mM})^{\mathrm{a})}$ & \\
\hline
\end{tabular}

a) These conditions were used for comparison of inhibitory activity among variants.

aligned using the BLAST 2 Sequences (BL2SEQ) program, and structurally conserved regions (SCRs) were configured and coordinated using the program Homology (Accelrys Software Inc., USA) to superimpose the threedimensional structure of the Kunitz protease inhibitor domain of protease nexin 2 (KPI) complexed with human factor XIa (PDB ID 1ZJD). Insertion regions were optimized with Insight II. Models of the triple mutant-factor $\mathrm{Xa}$ and leukocyte elastase complexes were constructed by superimposing the factor Xa (PDB ID 1IOE) and leukocyte elastase structures (PDB ID 1HNE) onto the factor XIa complex structure, respectively. Multiple sequence alignments of factor XIa, factor Xa, and leukocyte elastase were performed using ClustalW. SCRs of serine proteases were determined from the sequence analysis of various serine proteases. Superimpositions were performed using the Homology program. Each structural optimization was performed by molecular mechanics (MM) calculations with CVFF using Discover 3 (Accelrys Software Inc., USA). The structure and position of the main chain atoms within SCRs were maintained in all steps. First, side-chains within the variable regions (VRs) and all hydrogen atoms were optimized. In the resulting models, all atoms except backbone atoms within the SCRs were energy minimized in a similar manner. Finally, all hydrogen atoms were minimized and the optimized complex models were obtained. Residue positions were numbered from the mature $\mathrm{N}$-terminal isoleucine of human factor XIa, factor Xa, and leukocyte elastase.

\section{RESULTS AND DISCUSSION}

\subsection{Selection of Amino Acids for Introduction of a Third Mutation in Silico}

Bikunin is classified as a Kunitz-type protease inhibitor $[9,11]$. It is well known that the selectivity and potency of Kunitz-type protease inhibitors are attributed to an amino acid residue in the reactive center P1 that binds to the sub-site S1 of serine proteases. Arginine 15 in the second domain of bikunin corresponds to the P1 residue (Figure 1) [14]. Kunitz-type protease inhibitors with arginine or lysine at the $\mathrm{P} 1$ site and with alanine or valine at the P1 site prefer trypsin-like proteases including factor $\mathrm{Xa}$ and elastase-like proteases, respectively [9]. In our preliminary study on the second domain of bikunin, replacing arginine 15 with leucine drastically increased the leukocyte elastase inhibitory activity. However, this amino acid replacement abolished the inhibitory activeties against factor Xa and trypsin (data not shown). To potentiate the leukocyte elastase inhibitory activity of the second domain of bikunin with similar or greater inhibitory activity against procoagulant proteases including factor $\mathrm{Xa}$, we tried to select a candidate amino acid, excluding the P1 site, in the second domain of bikunin for modification.

To identify the key interactions between the second domain of bikunin and the human leukocyte elastase catalytic domain, we docked the modeled structures of the second domain of bikunin and human leukocyte elastase (data not shown). Based on the observed interacttions, steric hindrance and electrostatic repulsion were predicted between the side chains of arginine 11 of the second domain of bikunin and arginine 134 (numbered from the mature N-terminal isoleucine) of human leukocyte elastase. Therefore, we selected arginine 11 of the second domain of bikunin as the site for a third mutation.

\subsection{Preparation of Variants and Comparison of Leukocyte Elastase Inhibitory Activity}

Arginine 11 of the second domain of bikunin is a sterically bulky and positively charged P5 residue. We predicted that this residue would raise steric hindrance and electric repulsion at arginine 134 on the leukocyte elastase surface. Additionally, it was previously reported that positively charged amino acids, lysine and arginine, are distributed on the surface of human leukocyte elastase 
[22]. Therefore, we hypothesized that an acidic or hydrophilic amino acid would be a suitable substitution for arginine 11 in the second domain of bikunin to enhance a beneficial electrostatic interaction with the leukocyte elastase surface. To verify this hypothesis, six amino acids with different hydrophilicity, hydrophobicity, charge, and steric bulkiness were examined.

Using site-directed mutagenesis, we substituted arginine 11 of $\mathrm{D} 2(\mathrm{Q} 19 \mathrm{~K} / \mathrm{Y} 46 \mathrm{D})$ with glutamine, aspartate, asparagine, leucine, serine, and alanine (Figure 1), designated D2(R11Q/Q19K/Y46D), D2(R11D/Q19K/Y46D), D2(R11N/Q19K/Y46D), D2(R11L/Q19K/Y46D), D2(R 11S/Q19K/Y46D), and D2(R11A/Q19K/Y46D), respectively. These variant proteins were prepared and the purity was ascertained by SDS-PAGE. The inhibitory activity of each variant against human leukocyte elastase was expressed as an $\mathrm{IC}_{50}$ eduction relative to the $\mathrm{IC}_{50}$ of D2(Q19K/Y46D) (Table 2). Except for a leucine substitution, mutating arginine 11 enhanced the inhibitory activity against leukocyte elastase. A substitution by serine, which is a hydrophilic and small amino acid, enhanced the leukocyte elastase inhibitory activity about 7-fold compared to $\mathrm{D} 2(\mathrm{Q} 19 \mathrm{~K} / \mathrm{Y} 46 \mathrm{D})$. These results support our hypothesis. Then we selected D2(R11S/Q19K/Y46D) as the most potent triple point mutant.

\subsection{Inhibitory Activity of D2(R11S/Q19K/Y46D) against Various Serine Proteases}

The ability of D2(R11S/Q19K/Y46D) to inhibit procoagulant, proinflammatory, anticoagulant, and fibrinolytic serine proteases such as factor XIa, factor Xa, thrombin, factor VIIa, factor IXa, factor XIIa, leukocyte elastase, cathepsin G, plasma kallikrein, activated protein $\mathrm{C}$, tissue-type plasminogen activator (t-PA), urokinasetype plasminogen activator (u-PA), and plasmin were examined using chromogenic substrates and purified enzymes. D2(R11S/Q19K/Y46D) concentration-dependently inhibited factor XIa (Figure 2(a)), factor Xa (Figure 2(b)), and leukocyte elastase (Figure 2(c)) with $\mathrm{IC}_{50}$ values of 182,302 , and $273 \mathrm{nM}$, respectively. It also

Table 2. $\mathrm{IC}_{50}$ reductions of single amino acid substitution variants of $\mathrm{D} 2(\mathrm{Q} 19 \mathrm{~K} / \mathrm{Y} 46 \mathrm{D})$ relative to the $\mathrm{IC}_{50}$ of D2 (Q19K/Y46D) for human leukocyte elastase.

\begin{tabular}{cc}
\hline Variant & $\begin{array}{c}\text { Relative reduction in } \mathrm{IC}_{50} \text { to } \\
\mathrm{D} 2(\mathrm{Q} 19 \mathrm{~K} / \mathrm{Y} 46 \mathrm{D})\end{array}$ \\
\hline $\mathrm{D} 2(\mathrm{Q} 19 \mathrm{~K} / \mathrm{Y} 46 \mathrm{D})$ & 1.0 \\
$\mathrm{D} 2(\mathrm{R} 11 \mathrm{Q} / \mathrm{Q} 19 \mathrm{~K} / \mathrm{Y} 46 \mathrm{D})$ & 2.6 \\
$\mathrm{D} 2(\mathrm{R} 11 \mathrm{D} / \mathrm{Q} 19 \mathrm{~K} / \mathrm{Y} 46 \mathrm{D})$ & 1.3 \\
$\mathrm{D} 2(\mathrm{R} 11 \mathrm{~N} / \mathrm{Q} 19 \mathrm{~K} / \mathrm{Y} 46 \mathrm{D})$ & 3.0 \\
$\mathrm{D} 2(\mathrm{R} 11 \mathrm{~L} / \mathrm{Q} 19 \mathrm{~K} / \mathrm{Y} 46 \mathrm{D})$ & 1.0 \\
$\mathrm{D} 2(\mathrm{R} 11 \mathrm{~S} / \mathrm{Q} 19 \mathrm{~K} / \mathrm{Y} 46 \mathrm{D})$ & 7.4 \\
$\mathrm{D} 2(\mathrm{R} 11 \mathrm{~A} / \mathrm{Q} 19 \mathrm{~K} / \mathrm{Y} 46 \mathrm{D})$ & 2.4 \\
\hline
\end{tabular}

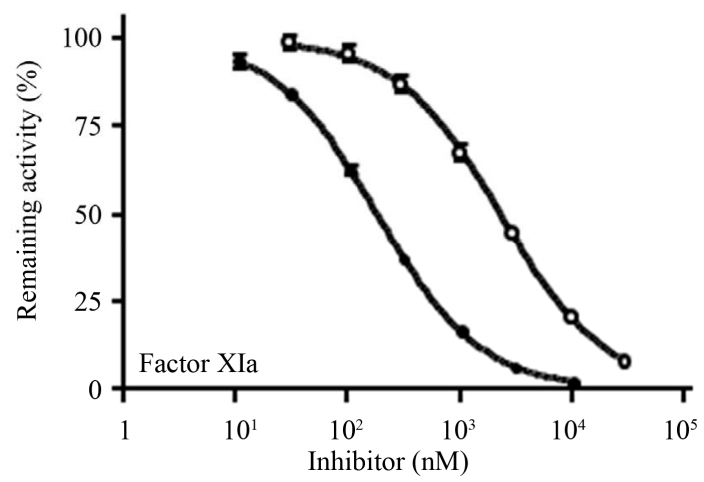

(a)

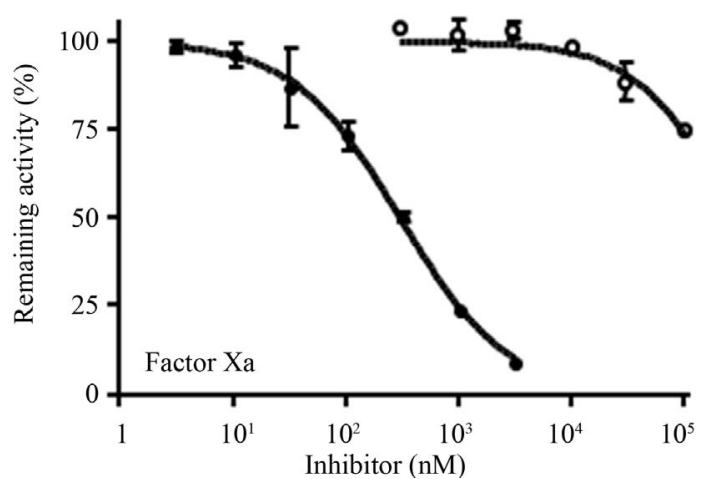

(b)

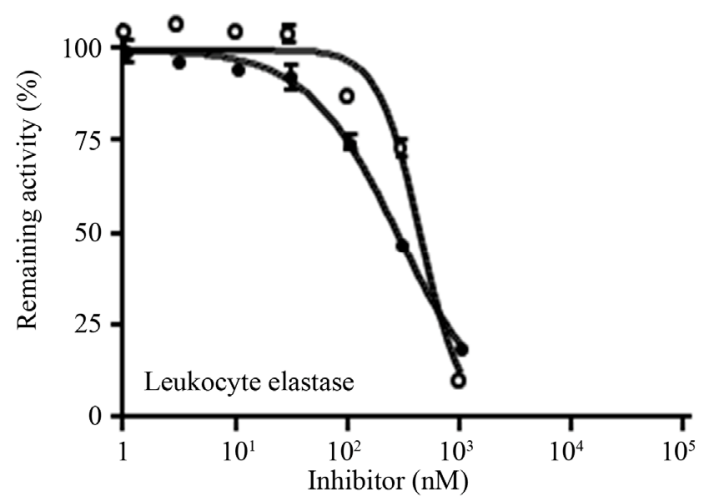

(c)

Figure 2. Inhibition of human factor XIa, factor Xa, and leukocyte elastase by D2(R11S/Q19K/Y46D) and bikunin. (a) Human factor XIa $(0.02 \mathrm{U} / \mathrm{mL})$ was preincubated at $37^{\circ} \mathrm{C}$ for $10 \mathrm{~min}$ with $\mathrm{D} 2(\mathrm{R} 11 \mathrm{~S} / \mathrm{Q} 19 \mathrm{~K} /$ Y46D) $(\bullet)$ or bikunin (०), added with the substrate $\mathrm{S}-2366(0.5 \mathrm{mM})$, and incubated at $37^{\circ} \mathrm{C}$ for $30 \mathrm{~min}$; (b) Human factor $\mathrm{Xa}(0.02 \mathrm{U} / \mathrm{mL})$ was pre-incubated at $37^{\circ} \mathrm{C}$ for $10 \mathrm{~min}$ with $\mathrm{D} 2(\mathrm{R} 11 \mathrm{~S} / \mathrm{Q} 19 \mathrm{~K} / \mathrm{Y} 46 \mathrm{D})(\bullet)$ or bikunin (०), added with the substrate S-2222 (0.4 mM), and incubated at $37^{\circ} \mathrm{C}$ for $30 \mathrm{~min}$; (c) Human leukocyte elastase $(2 \mu \mathrm{g} / \mathrm{mL})$ was pre-incubated at $37^{\circ} \mathrm{C}$ for $5 \mathrm{~min}$ with D2(R11S/Q19K/Y46D) (•) or bikunin (०), added with the substrate S-2484 $(0.2 \mathrm{mM})$, and incubated at $37^{\circ} \mathrm{C}$ for $5 \mathrm{~min}$. The reaction was stopped by the addition of acetate solution. Absorbance at $405 \mathrm{~nm}$ was measured to calculate enzyme activities. Data are expressed as the means and standard deviations of duplicate measurements. 
modestly inhibited plasmin with an $\mathrm{IC}_{50}$ value of 1340 nM (Table 3). On the other hand, D2(R11S/Q19K/Y46D) did not inhibit thrombin, factor VIIa, factor IXa, factor XIIa, cathepsin G, plasma kallikrein, activated protein C, t-PA, and u-PA even at 3130 to $10400 \mathrm{nM}$. In contrast, bikunin inhibited factor XIa (Figure 2(a)), leukocyte elastase (Figure 2(c)), and plasmin with $\mathrm{IC}_{50}$ values of 2300,433 , and $1140 \mathrm{nM}$, respectively, but did not inhibit factor Xa (Figure 2(b)), thrombin, factor VIIa, factor IXa, factor XIIa, cathepsin G, plasma kallikrein, activated protein $\mathrm{C}$, t-PA, and $\mathrm{u}-\mathrm{PA}$ even at 3000 to 100000 nM (Table 3). Thus, compared to bikunin, D2(R11S/Q19K/ Y46D) was a more potent inhibitor of factor XIa and factor Xa. Furthermore, leukocyte elastase inhibitory activeties of D2(R11S/Q19K/Y46D) and bikunin were comparable.

\subsection{Anticoagulant Activity in Human Plasma}

We expected that D2(R11S/Q19K/Y46D) could exert anticoagulant effects in plasma because of its inhibitory activities against factor Xa and factor XIa. Therefore, the effects of D2(R11S/Q19K/Y46D) on the activated partial thrombin time in human plasma were investigated and compared to those of bikunin. D2(R11S/Q19K/Y46D) dose-dependently prolonged activated partial thromboplastin time (Figure 3). The D2(R11S/Q19K/Y46D) concentration required to prolong the activated partial thromboplastin time 1.5 -fold was $0.82 \mu \mathrm{M}$. In contrast, bikunin only slightly prolonged the activated partial throm- bin time, which did not increase 1.5 -fold even at $8.3 \mu \mathrm{M}$. This results is consistent with the previous findings that the second domain of bikunin and D2(Q19K/Y46D) had anticoagulant activities in human plasma $[13,14]$. These results indicate that $\mathrm{D} 2(\mathrm{R} 11 \mathrm{~S} / \mathrm{Q} 19 \mathrm{~K} / \mathrm{Y} 46 \mathrm{D})$ has more

Table 3. $\mathrm{IC}_{50}$ values of bikunin and D2(R11S/Q19K/Y46D) for serine proteases.

\begin{tabular}{|c|c|c|c|}
\hline & \multirow[b]{2}{*}{ Enzyme } & \multicolumn{2}{|c|}{$\mathrm{IC}_{50}(\mathrm{nM})$} \\
\hline & & Bikunin & $\begin{array}{c}\text { D2(R11S/ } \\
\text { Q19K/Y46D) }\end{array}$ \\
\hline \multirow{6}{*}{ Procoagulant } & Factor XIa & 2300 & 182 \\
\hline & Factor Xa & $>100000$ & 302 \\
\hline & Thrombin (factor IIa) & $>10000$ & $>10400$ \\
\hline & Factor VIIa & $>10000$ & $>10400$ \\
\hline & Factor IXa & $>10000$ & $>10400$ \\
\hline & Factor XIIa & $>10000$ & $>10400$ \\
\hline \multirow{3}{*}{ Proinflammatory } & Leukocyte elastase & 433 & 273 \\
\hline & Cathepsin G & $>3000$ & $>3130$ \\
\hline & Plasma kallikrein & $>10000$ & 3590 \\
\hline \multirow[t]{2}{*}{ Anticoagulant } & Activated protein $\mathrm{C}$ & $>10000$ & $>10400$ \\
\hline & $\mathrm{t}-\mathrm{PA}$ & $>10000$ & $>10400$ \\
\hline \multirow[t]{2}{*}{ Fibrinolytic } & $\mathrm{u}-\mathrm{PA}$ & $>10000$ & $>10400$ \\
\hline & Plasmin & 1140 & 1340 \\
\hline
\end{tabular}

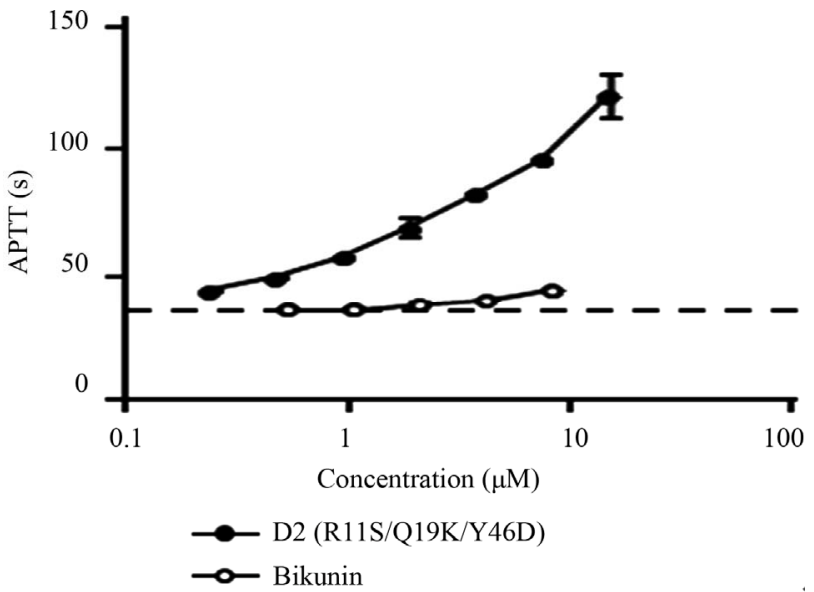

Figure 3. Effects of D2(R11S/Q19K/Y46D) and bikunin on activated partial thromboplastin time (APTT) in plasma. D2 $(\mathrm{R} 11 \mathrm{~S} / \mathrm{Q} 19 \mathrm{~K} / \mathrm{Y} 46 \mathrm{D})(\bullet)$ or bikunin $(\mathrm{O})$ was added to human plasma and APTT reagent was added to initiate clotting. Clotting time was measured using an automatic coagulometer. Data are expressed as the means and standard deviations of duplicate measurements. The dotted line indicates APTT in control plasma.

potent anticoagulant activity against intrinsic coagulation pathway than bikunin.

It has been proposed that the intrinsic pathway plays an important accessory role as an amplification loop leading to the expansion of intravascular thrombus volume once the tissue factor pathway activates coagulation $[3,6]$. In this study, we found that D2(R11S/Q19K/Y46D) inhibited factor XIa as well as factor Xa, which are known players in the amplification loop. This suggests that D2 (R11S/Q19K/Y46D) can prevent procoagulant responses during the expansion phase of pathological thrombogenesis.

\subsection{Inhibition of Leukocyte Elastase-Induced Endothelial Injury}

We also examined the inhibitory effect of D2(R11S/ Q19K/Y46D) on leukocyte elastase-induced endothelial cell injury to estimate its inhibitory effect on leukocyte elastase-induced degradation of a natural substrate. The viability of human umbilical vein endothelial cells was reduced by the addition of human leukocyte elastase. D2(R11S/Q19K/Y46D) moderately suppressed the reduction of elastase-induced cell viability at $0.15-15 \mu \mathrm{M}$ and was comparable to bikunin (Figure 4). This result is consistent with the previous observation that bikunin inhibited leukocyte elastase-induced endothelial cell injury [23] and indicates that D2(R11S/Q19K/Y46D) moderately protects endothelial cells from leukocyte elastase-induced injury as well as bikunin. Taken together with anticoagulant activity in human plasma, our results indicate that D2(R11S/Q19K/Y46D) not only can reduce hypercoagulability but also may protect endothelial cells from leukocyte elastase-induced damage. 


\subsection{Interactions between D2(R11S/Q19K/Y46D) and Serine Proteases}

D2(R11S/Q19K/Y46D) inhibited factor XIa and Xa as well as leukocyte elastase. Therefore, we modeled the docked structures of this novel variant with factor XIa, factor Xa, and leukocyte elastase using the X-ray crystal structure of the Kunitz protease inhibitor domain of protease nexin 2 complexed with factor XIa as a template [24]. D2(R11S/Q19K/Y46D) and the Kunitz protease inhibitor domain of protease nexin 2 (KPI) are highly similar with approximately $50 \%$ sequence identity (Figure 5). A homology model for $\mathrm{D} 2(\mathrm{R} 11 \mathrm{~S} / \mathrm{Q} 19 \mathrm{~K} / \mathrm{Y} 46 \mathrm{D})$ was built by coordinating with the SCRs of the Kunitz protease inhibitor domain complexed with human factor XIa. Each protease structure was constructed based on the results of multiple sequence alignments (Figure 6). We built three models of D2(R11S/Q19K/Y46D)-protease complexes by aligning $\mathrm{D} 2(\mathrm{R} 11 \mathrm{~S} / \mathrm{Q} 19 \mathrm{~K} / \mathrm{Y} 46 \mathrm{D})$ and the proteases onto the co-crystal structure of the Kunitz protease inhibitor domain and factor XIa. These new models suggested that the three mutations affected interactions between D2(R11S/Q19K/Y46D) and the proteases. In

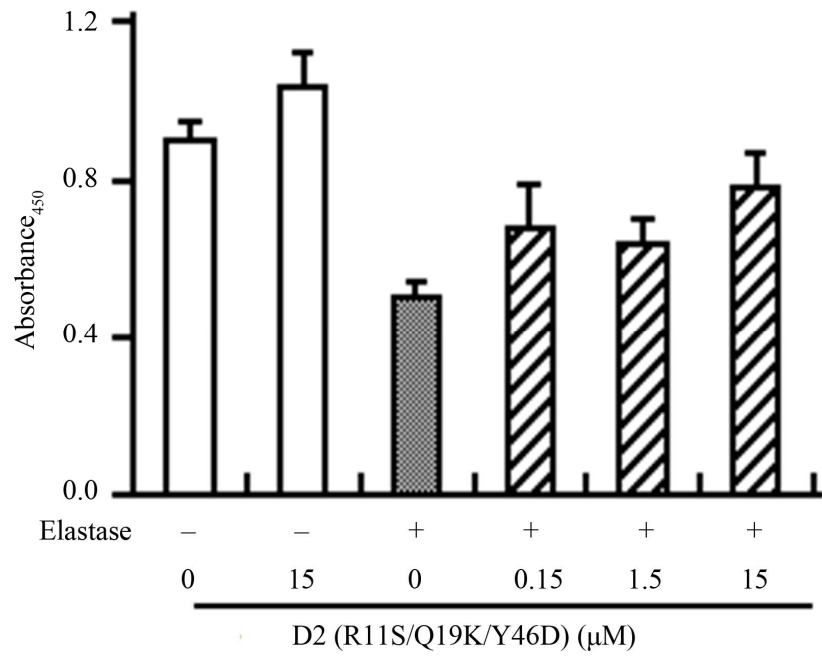

(a)
D2(R11S/Q19K/Y46D), lysine 19 and aspartate 46 were predicted to form an intramolecular salt bridge. In the variant-factor XIa complex, it was predicted that serine 11 of D2(R11S/Q19K/Y46D) would avoid electrostatic repulsion with the positive charge on lysine 182 in factor XIa and formed a hydrogen bond with lysine 182 in factor XIa. In addition, lysine 19 of D2(R11S/Q19K/Y46D) was predicted to form a hydrogen bond with glycine 46 in factor XIa (Figure 7(a)). In the variant-factor Xa complex, lysine 19 of D2(R11S/Q19K/Y46D) was predicted to form hydrogen bonds with glutamate 21 and glutamate 22 in factor Xa. Aspartate 46 of D2(R11S/ Q19K/Y46D) was also predicted to form a hydrogen bond with lysine 48 in factor Xa (Figure 7(b)). In the variant-leukocyte elastase complex, it was predicted that serine 11 of D2(R11S/Q19K/Y46D) would not repulse the positive charge on arginine 134 in leukocyte elastase. This prediction was supported by the present result that D2(R11S/Q19K/Y46D) exhibited the more potent leukocyte elastase inhibitory activity compared to D2 (Q19K/Y46D). Lysine 19 of D2(R11S/Q19K/Y46D) was predicted to form a hydrogen bond with asparagine 48 in leukocyte elastase. Hydrogen bonds between aspartate 46 in D2(R11S/Q19K/Y46D) and asparagine 46 in leukocyte

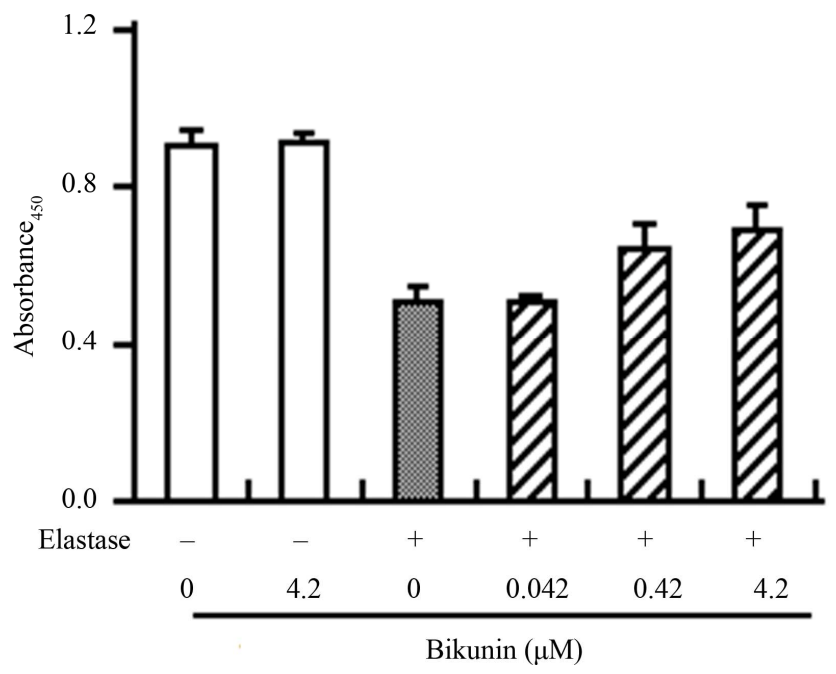

(b)

Figure 4. Effects of D2(R11S/Q19K/Y46D) and bikunin on human leukocyte elastase-induced endothelial cell injury in human umbilical vein endothelial cells. Endothelial cells were incubated with D2(R11S/Q19K/Y46D) (a) and bikunin (b) in the presence or absence of purified elastase $(0.3 \mu \mathrm{g} / \mathrm{mL})$ for $2 \mathrm{~h}$. Cell viability was determined by the absorbance at $450 \mathrm{~nm}$ using WST-1 reagent. Each column represents the mean and standard deviation of triplicate measurements.

KPI

D2 (R11S/Q19K/Y46D) --AACNLPIVSGPCRAFIKLWAFDAVKGKCVLFPYGGCQGNGNKFDSEKECREYCGVPGDGDEELLRFSN

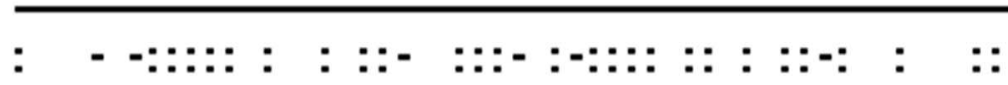

Figure 5. Local alignment of the amino acid sequences of the Kunitz protease inhibitor domain of protease nexin 2 (KPI) and D2(R11S/Q19K/Y46D). The structurally conserved regions (SCRs) for homology modeling templates are underlined. Identical and related amino acid residues are indicated under the alignments with : and -, respectively. 
Factor XIa

Factor $\mathrm{Xa}$

Leukocyte elastase
Factor XIa
Factor Xa
Leukocyte elastase

Factor XIa

Factor $\mathrm{Xa}$

Leukocyte elastase $\begin{array}{llll}10 & 20 & 30 & 40 \\ \text { IVGGTASVR-GEWPWQVTLHTTSPTQRHLCGGSI I GNQWILTAAHCFYGVE-SPKILRVY } \\ \text { IVGGQEC-KDGECPWQALL-INEENEG-FCGGTILSEFYILTAAHCLYQAK-RFKV-RV- } \\ \text { IVGGRRA-RPHAWPFMVSL---QLRGGHFCGATLI IAPNFVMSAAHCVANVNVR-AV-RVV }\end{array}$

60

60

70

80

90

100

SGILNQAEIKED--T-SFFGVQEI I I-HDQYKMAES-GYDIALLKLE-T-TVNY-ADSQR -GDRN-TE-QEEGGE-AVHEV-EVVIKHNRFT-KETYDFDIAVLRLK-T-PITFRMNVALGAHNLSR-REP--TRQVFAV-QRIF-EDGYDPVNLLN-DIVILQLNGSATIN--ANVQ-

\begin{tabular}{l|l|l|l|}
110 & 120 & 130 & 140 \\
| & $\mid$ & $\mid$
\end{tabular}

160

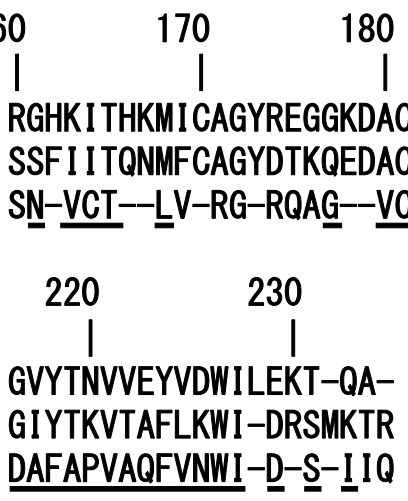

Figure 6. Multiple alignments of human factor XIa, human factor Xa, and human leukocyte elastase amino acid sequences. The structurally conserved regions (SCRs) for superimposition are underlined. Residue positions are numbered from the mature N-terminus of each enzyme.

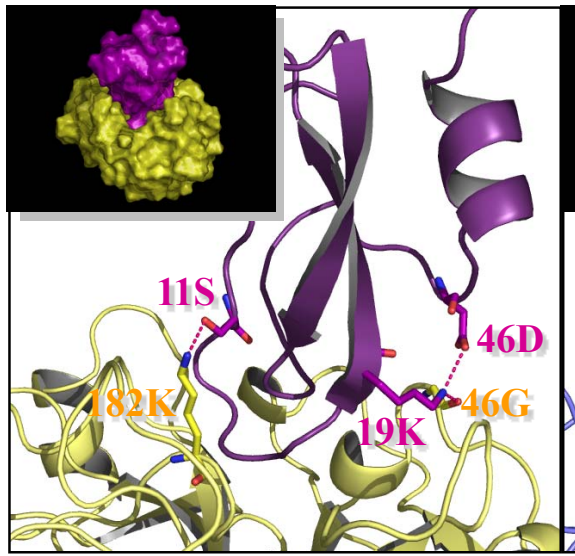

(a)

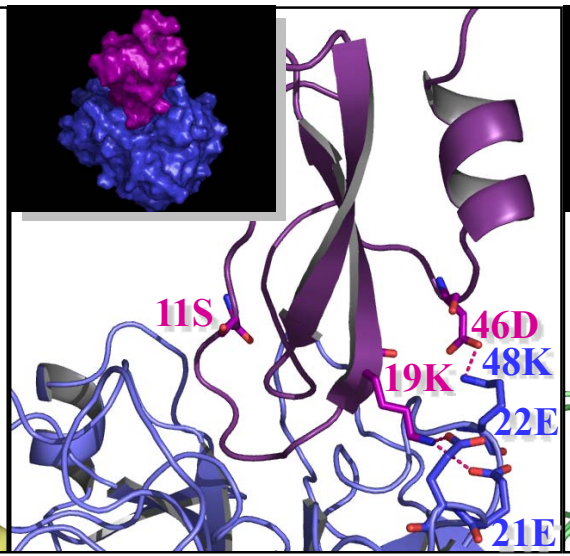

(b)

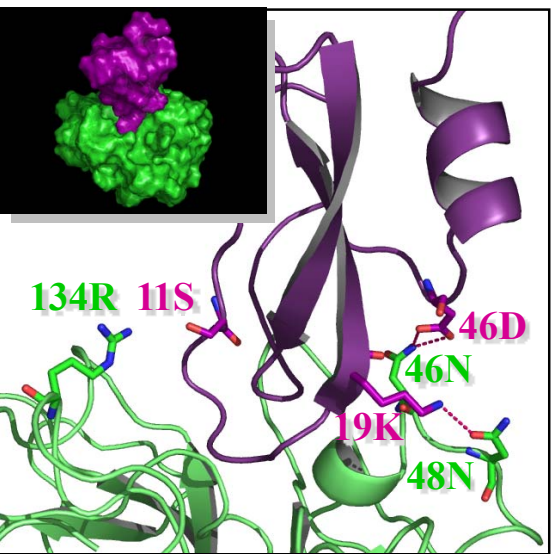

(c)

Figure 7. Models of D2(R11S/Q19K/Y46D) complexed with factor XIa, factor Xa, and leukocyte elastase. Factor XIa (a), factor Xa (b), and leukocyte elastase (c) are colored yellow, blue, and green, respectively. D2(R11S/Q19K/Y46D) is colored purple. Nitrogen and oxygen atoms are colored blue and red, respectively. Hydrogen bonds are indicated with red dotted lines. An intramolecular salt bridge in D2(R11S/Q19K/Y46D) is also indicated with red dotted lines. Residue positions are numbered from the mature N-terminus of each enzyme. The figures were drawn using the molecular graphics software PyMol v0.99 (DeLano Scientific, USA). 
elastase were also predicted (Figure 7(c)). We speculated that these favorable interactions might contribute to the potent inhibitory activities against factor XIa, factor Xa, and leukocyte elastase.

\section{CONCLUSION}

Using computer modeling and site-directed mutagenesis, we generated a novel variant of the second domain of bikunin with arginine 11, glutamine 19, and tyrosine 46 substituted to serine, lysine, and aspartate, respectively, that has potent inhibitory activities against factor XIa, factor $\mathrm{Xa}$, and leukocyte elastase. This novel triple mutated variant may have possible protective effects against hypercoagulation and endothelial injury.

\section{ACKNOWLEDGEMENTS}

We thank Keiko Hoshida, Tamami Ono, Tsubasa Fujii, and Yuhiro Sakakibara for their excellent technical assistance. We also wish to thank Jiro Hirose, Toru Yamakawa, and Tomokazu Matsusue for their helpful suggestions and great assistance in developing this research and document.

\section{REFERENCES}

[1] Esmon, C.T. (2003) Inflammation and thrombosis. Journal of Thrombosis and Haemostasis, 1, 1343-1348. http://dx.doi.org/10.1046/j.1538-7836.2003.00261.x

[2] Mann, K.G. (1999) Biochemistry and physiology of blood coagulation. Journal of Thrombosis and Haemostasis, 82, 165-174.

[3] Gailani, D. and Renne, T. (2007) The intrinsic pathway of coagulation: A target for treating thromboembolic disease? Journal of Thrombosis and Haemostasis, 5, 1106-1112. http://dx.doi.org/10.1111/j.1538-7836.2007.02446.x

[4] Tucker, E.I., Gailani, D., Hurst, S., Cheng, Q., Hanson, S.R. and Gruber, A. (2008) Survival advantage of coagulation factor XI-deficient mice during peritoneal sepsis. Journal of Infectious Diseases, 198, 271-274. http://dx.doi.org/10.1086/589514

[5] Martorell, L., Martinez-Gonzalez, J., Rodriguez, C., Gentile, M., Calvayrac, O. and Badimon, L. (2008) Thrombin and protease-activated receptors (PARs) in atherothrombosis. Journal of Thrombosis and Haemostasis, 99, 305-315.

[6] Opal, S.M. and Esmon, C.T. (2003) Bench-to-bedside review: Functional relationships between coagulation and the innate immune response and their respective roles in the pathogenesis of sepsis. Critical Care, 7, 23-38. http://dx.doi.org/10.1186/cc1854

[7] Lee, W.L. and Downey, G.P. (2001) Leukocyte elastase: Physiological functions and role in acute lung injury. American Journal of Respiratory and Critical Care Medicine, 164, 896-904.

[8] Hirose, J., Ozawa, T., Miura, T., Isaji, M., Nagao, Y., Yamashiro, K., Nii, A., Kato, K. and Uemura, A. (1998) Human neutrophil elastase degrades inter-alpha-trypsin in- hibitor to liberate urinary trypsin inhibitor related proteins. Biological and Pharmaceutical Bulletin, 21, 651-656. http://dx.doi.org/10.1248/bpb.21.651

[9] Pugia, M.J. and Lott, J.A. (2005) Pathophysiology and diagnostic value of urinary trypsin inhibitors. Clinical Chemistry and Laboratory Medicine, 43, 1-16. http://dx.doi.org/10.1515/CCLM.2005.001

[10] Fries, E. and Kaczmarczyk, A. (2003) Inter-alpha-inhibitor, hyaluronan and inflammation. Acta Biochimica Polonica, 50, 735-742.

[11] Fries, E. and Blom, A.M. (2000) Bikunin-Not just a plasma proteinase inhibitor. The International Journal of Biochemistry \& Cell Biology, 32, 125-137. http://dx.doi.org/10.1016/S1357-2725(99)00125-9

[12] Xu, Y., Carr, P.D., Guss, J.M. and Ollis, D.M. (1998) The crystal structure of bikunin from the inter-alpha-inhibitor complex: A serine protease inhibitor with two Kunitz domains. Journal of Molecular Biology, 276, 955-966. http://dx.doi.org/10.1006/jmbi.1997.1582

[13] Morishita, H., Yamakawa, T., Matsusue, T., Kusuyama, T., Sameshima-Aruga, R., Hirose, J., Nii, A., Miura, T., Isaji, M., Horisawa-Nakano, R., Nagase, Y., Kanamori, T., Nobuhara, M., Tanaka, R., Koyama, S. and Naotsuka, M. (1994) Novel factor Xa and plasma kallikrein inhibittory-activities of the second Kunitz-type inhibitory domain of urinary trypsin inhibitor. Thrombosis Research, 73, 193-204. http://dx.doi.org/10.1016/0049-3848(94)90098-1

[14] Nii, A., Morishita, H., Yamakawa, T., Matsusue, T., Hirose, J., Miura, T., Isaji, M., Horisawa, Y., Sugihara, K., Kanamori, T. and Umeyama, H. (1994) Design of variants of the second domain of urinary trypsin inhibitor (R-020) with increased factor Xa inhibitory activity. Journal of Biochemistry, 115, 1107-1112.

[15] Creighton, T.E. (1992) Proteins: Structures and molecular properties. W. H. Freeman and Company, New York, 253255.

[16] Rawlings, N.D. and Barrett, A.J. (1994) Families of serine peptidases. In: Barrett, A.J., Ed., Methods in Enzymology, Academic Press, San Diego, 19-61.

[17] Powers, J.C., Kam, C.M., Narasimhan, L., Oleksyszyn, J., Hernandez, M.A. and Ueda, T. (1989) Mechanism-based isocoumarin inhibitors for serine proteases: Use of active site structure and substrate specificity in inhibitor design. Journal of Cellular Biochemistry, 39, 33-46. http://dx.doi.org/10.1002/jcb.240390105

[18] Marquart, M., Walter, J., Deisenhofer, J., Bode, W. and Huber, R. (1983) The geometry of the reactive site and of the peptide groups in trypsin, trypsinogen and its complexes with inhibitors. Acta Crystallographica Section B, 39, 480-490. http://dx.doi.org/10.1107/S010876818300275X

[19] Miyata, T., Sakai, T., Sugimoto, M., Naka, H., Yamamoto, K., Yoshioka, A., Fukui, H., Mitsui, K., Kamiya, K., Umeyama, H. and Iwanaga, S. (1991) Factor IX Amagasaki: A new mutation in the catalytic domain resulting in the loss of both coagulant and esterase activities. Biochemistry, 30, 11286-11291. http://dx.doi.org/10.1021/bi00111a014

[20] Landt, O., Grunert, H.P. and Hahn, U. (1990) A general 
method for rapid site-directed mutagenesis using the polymerase chain reaction. Gene, 96, 125-128. http://dx.doi.org/10.1016/0378-1119(90)90351-Q

[21] Messing, J. and Vieira, J. (1982) A new pair of M13 vectors for selecting either DNA strand of double-digest restriction fragments. Gene, 19, 269-276. http://dx.doi.org/10.1016/0378-1119(82)90016-6

[22] Navia, M.A., McKeever, B.M., Springer, J.P., Lin, T.Y., Williams, H.R., Fluder, E.M., Dorn, C.P. and Hoogsteen K. (1989) Structure of human neutrophil elastase in complex with a peptide chloromethyl ketone inhibitor at 1.84A resolution. PNAS, 86, 7-11. http://dx.doi.org/10.1073/pnas.86.1.7

[23] Nakatani, K., Takeshita, S., Tsujimoto, H., Kawamura, Y. and Sekine, I. (2001) Inhibitory effect of serine protease inhibitors on neutrophil-mediated endothelial cell injury. Journal of Leukocyte Biology, 69, 241-247.

[24] Navaneetham, D., Jin, L., Pandey, P., Strickler, J.E., Babine, R.E., Abdel-Meguid, S.S. and Walsh, P.N. (2005) Structural and mutational analyses of the molecular interactions between the catalytic domain of factor XIa and the Kunitz protease inhibitor domain of protease nexin 2. Journal of Biological Chemistry, 280, 36165-36175. http://dx.doi.org/10.1074/jbc.M504990200 